\title{
Open-Source Hardware Is a Low-Cost Alternative for Scientific Instrumentation and Research
}

\author{
Daniel K. Fisher ${ }^{1}$, Peter J. Gould ${ }^{2}$ \\ ${ }^{1}$ USDA Agricultural Research Service, Stoneville, USA \\ ${ }^{2}$ US Forest Service, Pacific Northwest Research Station, Olympia, USA \\ Email: daniel.fisher@ars.usda.gov, pgould@fs.fed.us
}

Received January 29, 2012; revised February 28, 2012; accepted March 9, 2012

\begin{abstract}
Scientific research requires the collection of data in order to study, monitor, analyze, describe, or understand a particular process or event. Data collection efforts are often a compromise: manual measurements can be time-consuming and labor-intensive, resulting in data being collected at a low frequency, while automating the data-collection process can reduce labor requirements and increase the frequency of measurements, but at the cost of added expense of electronic data-collecting instrumentation. Rapid advances in electronic technologies have resulted in a variety of new and inexpensive sensing, monitoring, and control capabilities which offer opportunities for implementation in agricultural and natural-resource research applications. An Open Source Hardware project called Arduino consists of a programmable microcontroller development platform, expansion capability through add-on boards, and a programming development environment for creating custom microcontroller software. All circuit-board and electronic component specifications, as well as the programming software, are open-source and freely available for anyone to use or modify. Inexpensive sensors and the Arduino development platform were used to develop several inexpensive, automated sensing and datalogging systems for use in agricultural and natural-resources related research projects. Systems were developed and implemented to monitor soil-moisture status of field crops for irrigation scheduling and crop-water use studies, to measure daily evaporation-pan water levels for quantifying evaporative demand, and to monitor environmental parameters under forested conditions. These studies demonstrate the usefulness of automated measurements, and offer guidance for other researchers in developing inexpensive sensing and monitoring systems to further their research.
\end{abstract}

Keywords: Open-Source Hardware; Arduino; Microcontrollers; Sensors; Datalogger

\section{Introduction}

Scientific research requires the collection of data in order to study, monitor, analyze, describe, or understand a particular process or event. Data collection efforts are often a compromise, however, between the amount and type of measurements needed and the resources available to collect them. Manual measurements can be time-consuming and labor-intensive, resulting in data being collected at a low frequency, with long time intervals between measurements. If outdoor field research is involved, collection intervals can be irregular when labor is unavailable, on weekends or when other duties take priority for example, or when inclement weather does not permit visits to the field. Automating the data-collection process can reduce labor requirements and greatly increase the frequency and regularity of measurements, but at the cost of added expense of electronic data-collecting instrumentation.

A vast number of electronic solutions are available for automated sensing, monitoring, and collecting information, but several problems exist which can limit their application in research work and acceptance by research scientists. Features, capabilities, and prices of commercially available datalogging instrumentation can vary greatly, from inexpensive, low-resolution, limited-input devices to expensive, full-featured, multi-input instruments. Developed by private industry, monitoring equipment often contains proprietary technology that manufacturers do not wish to release, and is often designed to operate with only a particular manufacturer's sensors. The user can become locked into a particular manufacturer's systems or sensor technology due to high costs of the monitoring equipment making it cost-prohibitive to switch to a different vendor. If a range of different sensor information is desired, a single vendor may not supply all that is needed, and several monitoring systems may be required due to incompatible technologies. Since the scientific data-collection and monitoring market is small, private companies may be slow to innovate or introduce new technologies based solely on economic analyses. And to obtain sufficient quantities of data from an ex- 
periment, multiple sites and replicated treatments may be needed to satisfy observational and statistical requirements, which can quickly become cost-prohibitive.

Rapid advances in electronic technologies have resulted in a variety of new and inexpensive sensing, monitoring, and control capabilities. These rapidly evolving technologies provide researchers and practitioners with access to low-cost, solid-state sensors and programmable microcontroller-based circuits. Microcontrollers can be thought of as small, low-power, low-cost computers packaged within a single chip. The microcontroller runs a program that is created and uploaded by the user to operate different components within a circuit. The user can modify the program and change the function of the circuit without changing the circuit physically. Many types of sensors and auxiliary components, such as memory chips, clocks, and communications devices, are available which interface directly with microcontrollers, simplifying circuit designs and putting electronic design within reach of people with limited electronics background and knowledge. A number of microcontroller-based devices have been described in which the specific requirements of a research project dictated the development of customized monitoring systems with unique capabilities [1-5].

A further advancement in microcontroller-based sensing and monitoring relates not specifically to the design and development of the electronics and physical components, but to the idea of making the designs and development efforts freely available to all in order to facilitate and expand the adoption of the technologies. The rapid rise of the internet and accessibility of computer resources led to the concept of Open Source Software as a means to provide free and transparent access to computer code so that individuals could review, modify, improve, and distribute computer software (Open Source Initiative, http://www.opensource.org). In recent years, a similar effort was undertaken to enable the free and open sharing of hardware designs and projects so that, by sharing and collaborating with others who have similar interests and needs, innovation could occur more quickly, improvements could be suggested and incorporated, and more users could access the final product.

One such Open Source Hardware project resulted in the creation of a microcontroller-based development platform called Arduino [6]. The Arduino hardware consists of a programmable microcontroller mounted on a circuit board which provides convenient access to the microcontroller input/output pins and connectivity to a personal computer for programming and user interaction. The circuit board has a standardized size and physical configuration so that any Arduino-compatible boards can be interchanged. Standardized add-on boards (called shields) plug into the Arduino circuit board, and are used to expand the capabilities of the main board. The microcontroller is programmed via the Arduino Integrated Development Environment (IDE), in which the user creates the program instructions to operate the microcontroller and then downloads the program to the microcontroller. As an open-source hardware project, all circuitboard and electronic component specifications, as well as the IDE software, are freely available for anyone to use or modify. As a result, private manufacturers all around the world produce and offer inexpensive, standardized Arduino-compatible hardware with an extensive supply of features and capabilities. Researchers have begun to develop and implement devices based on the Arduino platform for a variety of applications [7-12], with ease of use, low cost, and standardized components and programming language cited as reasons for choosing the Arduino platform.

The objective of this paper is to introduce researchers and practitioners to potential applications of the opensource Arduino platform for implementation in research and monitoring applications. Specifically, we 1) describe the Arduino microcontroller development platform, 2) discuss examples of sensing and auxiliary circuit components available, and 3) demonstrate several datalogging devices developed for use in agricultural and natural-resources research.

\section{Components}

\subsection{Arduino Microcontroller Development Platform}

The current standard Arduino development platform is based on an ATmega328 8-bit programmable microcontroller (Atmel Corporation, San Jose, CA USA). A printed-circuit board positions the microcontroller in a circuit so that the input/output (IO) pins are easily accessible. The microcontroller contains 32 kilobytes (KB) of flash memory for program storage and $1 \mathrm{~KB}$ of non-volatile data-storage memory. IO lines consist of 14 digital pins and 6 analog pins, which provide 6 channels of 10-bit analog-to-digital (A/D) conversion capability. The microcontroller contains many built-in features, including timer/counters, internal and external interrupts, serial and other communication-protocol capabilities, programmable watchdog timer, and low-power, energy-saving modes.

Versions of the Arduino board are available which use other, more-powerful microcontrollers, have additional IO pins, and have different physical sizes. Devices operate at either a $5-\mathrm{V}$ level and oscillator speed of $16 \mathrm{MHz}$ or a 3.3-V level and $8 \mathrm{MHz}$. While many boards have an on-board USB connector to interface with a personal computer, the ATmega microcontroller communicates via a two-wire serial (transmit, $\mathrm{Tx}$, and receive, $\mathrm{Rx}$ ) connection. Boards with on-board USB connector also 
have a USB-serial converter chip and use a standard USB-USB cable, while other boards, to simplify design and lower cost, do not incorporate the USB-serial chip. A special cable, which contains the USB-serial chip and creates a virtual serial port, must be used.

The Arduino board is designed to allow expansion through the connection of auxiliary boards or shields. The shields connect via mating pins which are arranged in the same physical configuration as the Arduino board, and simply plug onto the headers on the top of the Arduino board. The shields are then controlled by the Arduino microcontroller and program, which access the shields' pins through the Arduino pins. Programming libraries allow users to quickly integrate new devices and sensors into projects without needing to write extensive new program routines.

\subsection{Software}

The software environment for programming and interacting with the Arduino board is available for download and installation for several computer operating systems (GNU/Linux, Mac OS X, and Windows). Using the IDE, the user writes programs in a language based on $\mathrm{C}++$. The IDE then compiles and error-checks the program, and downloads the compiled routine to the microcontroller. A terminal window is available for outputting text and data from the Arduino board to the computer monitor and for interacting with the microcontroller.

As an open-source project, the Arduino benefits from the collective efforts and expertise of developers from around the world. Programming libraries, which contain routines to simplify programming and incorporate advanced features, sample code, and complete programs are available to download, use, and modify as needed. The IDE, libraries, and sample code can be accessed via the Arduino project website [6].

\subsection{Communications}

The Arduino development platform provides several methods of communicating with external components, sensors, and computers. In addition to built-in A/D converters and timers for measuring analog voltage signals, several standardized communications protocols are available for interfacing digital components and sensors.

The Inter-Integrated Circuit, also called $\mathrm{I}^{2} \mathrm{C}$ or $\mathrm{I} 2 \mathrm{C}$, protocol developed by Philips Semiconductor, is a twowire serial transfer protocol designed for communications between integrated-circuit chips and microcontrollers. Two IO pins on the Arduino's ATmega328 microcontroller are designated for $\mathrm{I} 2 \mathrm{C}$ communication. Each I2C device has its own unique identification number and address, allowing multiple devices to be connected to the same $\mathrm{I} 2 \mathrm{C}$ pins. The microcontroller initiates communica- tion with a device by first sending the address of the device and then reading data from or writing data to the device. Identification numbers are unique to each type of component (memory chip, clock, temperature sensor, etc.) while addresses are either preset by the manufacturer or specified by the user through different hardware configurations.

The Dallas 1-Wire protocol, developed by Dallas Semiconductor, uses a single IO pin for communication and, optionally, to power the external 1-Wire device. Like I2C, multiple devices can be connected to a single 1-Wire pin, and are called by the microcontroller using the device's unique address.

The Serial Peripheral Interface, or SPI, is a four-wire system developed by Motorola and provides a serial data link that operates in full duplex mode. SPI devices communicate in master/slave mode using three IO pins, with the master device, the microcontroller, initiating communications with the slave, a sensor or other device. The microcontroller uses an additional IO pin for each device to select and communicate with a particular device.

RS-232 is the standard serial communication protocol that was widely used to communicate between personal computers and peripherals before the advent of the universal serial bus (USB). RS-232 uses two communication lines ( $\mathrm{Rx}$ to receive, $\mathrm{Tx}$ to transmit), and is the protocol used by the Arduino' microcontroller to interface with a computer for programming. Since few modern computers contain an RS-232 port, a virtual serial port must be created. While some Arduino boards have a USB-to-serial converter chip on-board, many boards do not in order to reduce cost and power consumption. A special USBserial cable which contains the converter chip, such as the FTDI Cable (www.makerspace.com), interfaces to the computer's USB hub and creates a virtual serial port.

\subsection{Sensors}

A large number of sensors are available to monitor and measure many types of environmental parameters or physical processes. The rapid advances and usage of programmable microcontrollers have brought an increase in the availability and ease of use of sensing devices designed to interface with microcontrollers. The sensors operate at low voltages, and output signals compatible with microcontrollers, including analog voltages, varying frequencies, and a selection of digital communications protocols.

While the number of parameters sensed, and the number of sensors available, is vast, a few examples are presented and discussed in the following subsections.

\subsubsection{Temperature}

One of the most-common measurements made in a mul- 
titude of disciplines is temperature. A variety of temperature sensors is available using several different measurement technologies. While thermistors, which are sensors whose electrical resistance changes in response to temperature, are still in use, alternate electronic sensors are available which are designed to interface easily with microcontrollers and computers.

Analog temperature sensors, such as the LM35 (National Semiconductor, Santa Clara, CA USA) and TMP36 (Analog Devices, Inc, Norwood, MA USA), are designed to output a voltage signal proportional to temperature. The microcontroller supplies an excitation voltage to the sensor, and then measures the sensor's output voltage with an on-board A/D converter. The microcontroller program calculates temperature using a calibration developed by the sensor manufacturer. The LM35 sensor, for example, provides a linear response with a calibration of $10 \mathrm{mV} / \mathrm{C}$ : temperature $\left({ }^{\circ} \mathrm{C}\right)$, is therefore calculated by dividing the output voltage, in $\mathrm{mV}$, by 10. Analog sensors are usually very inexpensive and easy to work with, requiring only a simple voltage measurement and calibration equation to determine temperature. The microcontroller must have an $\mathrm{A} / \mathrm{D}$ converter, and a stable reference voltage, which some may not have, requiring the addition of external components and circuitry.

Digital temperature sensors are designed to provide a calibrated and voltage-converted output which can be read directly as a temperature value. These sensors do not require a voltage measurement to be made, allowing the use of microcontrollers which do not have A/D converters. Digital sensors interface with the microcontroller through one of several communications protocols, such as I2C, 1-Wire, and SPI, with transfer of information accomplished via the microcontroller program. Digital sensors often have the feature of a unique identification number, allowing multiple sensors to be connected to the same IO pins on the microcontroller, thus not using additional pins. In contrast, since each analog sensor would require its own $\mathrm{A} / \mathrm{D}$ input pin, multiple analog sensors could quickly fill available A/D converter pins.

For making non-contact temperature measurements, infrared thermometer (IRT) sensors are available which are inexpensive and easy to interface. The MLX90614 (Melexis SA, Ieper, Belgium) series of IRTs communicate with the microcontroller via the I2C protocol. Experience using these sensors to monitor crop canopy temperature [5] has shown them to work well in a harsh agricultural environment, operate for extended periods under battery power, and provide accurate temperature measurements.

\subsubsection{Soil-Water Status}

In many agricultural, natural-resource, and water-mana- gement disciplines, water availability and moisture status are of great importance. The amount of water available in the soil profile for extraction by growing plants can be measured with a water-content sensor. A water potential sensor provides a measure of how tightly the water is held to the soil particles and how much energy must be expended to extract the water by the plant roots. This can be related to the availability of water to the plant.

Many of the currently available water-content sensors rely on a measure of the capacitance of the soil-water environment. Dielectric properties of the soil-water system vary weakly with soil properties, such as mineral composition, bulk density, and organic-matter content, but are strongly influenced by water content [13]. Watercontent sensors, such as the EC-5 and EC-20 (Decagon Devices, Pullman, WA USA), and VG400 (Vegetronix, Bluffdale, UT USA), consist of a capacitive-sensing element and on-board electronic circuitry. When powered by the microcontroller, the sensors return a voltage signal proportional to the water content in the soil. Measuring the voltage with the microcontroller's A/D converter and applying a calibration equation in the microcontroller program results in a water-content value, expressed in units of volume of water/volume of soil. Sensor manufacturers may provide calibration equations for limited soil types and other porous media, such as potting soil or greenhouse media, but the user often must develop a calibration, or at least verify the manufacturer's, under his specific soil conditions to obtain accurate water-content measurements.

Water-potential sensors are usually designed to act as variable resistors, in which the electrical resistance of the sensor varies in response to its water content. The sensor is composed of a porous matrix, and water can move into and out of the matrix in response to the matric potential of the soil. As the water content in the porous matrix changes with matric potential, the electrical resistance also changes. A calibration equation then converts resistance to matric or water potential, expressed in units of kiloPascals $(\mathrm{kPa})$.

The Watermark 200SS (Irrometer Company, Riverside, CA USA) water-potential sensor is popular in irrigation-scheduling applications due to its ease of installation and low cost. It requires an alternating-current excitation rather than direct current, however, which can involve additional care when interfacing with a microcontroller (see [3] and the discussion in Section 3.1 below for alternative implementations). To allow direct connection and use with any microcontroller circuit, the MPS-2 (Decagon Devices, Pullman, WA USA) is designed to operate from a direct-current supply and output a simple voltage signal in response to soil-water potential. The voltage signal is measured with the microcontroller's $\mathrm{A} / \mathrm{D}$ converter and then converted to water potential with 
a calibration equation.

\subsubsection{Distance/Height}

Distance measurements are common in robotic and industrial/manufacturing environments to determine distance from a moving vehicle for obstacle avoidance, detect presence or absence of material, and ensure proper placement of a component. In research applications, distance measurements can be used to determine properties such as plant height and canopy width, depth of water in canals, and fluid levels in tanks.

Distance measurements are commonly made using two sensing technologies, ultrasonic and infrared. Ultrasonic sensors often consist of two transducers, one which emits a pulse of high-frequency sound waves, and a second one to detect the sound after reflecting off a nearby surface. Distance is determined by measuring the length of time between sending the pulse and receiving the reflection, or echo, and converting this to a distance based on the speed of sound. Ultrasonic sensors, such as the SRF series (Devantech Ltd., Norfolk, UK) and the PING (Parallax Inc., Rocklin, CA USA) interface with a microcontroller via one or two digital IO pins. The microcontroller is programmed to initiate a pulse, then starts an internal timer and counts the number of microseconds until an echo signal is detected, and calculates the distance based on this time interval. Sensors are available with varying fields of view to enable sensing over wider or narrower regions.

Infrared sensors operate by emitting a beam of light and detecting the reflected beam, after hitting an obstacle, with a light sensor. The reflected beam returns at a slight angle from the emitted beam, and the angle of the two beams is dependent on the distance of the obstacle from the sensor. The reflected beam strikes the light sensor at some point, and is read by an on-board microcontroller which is programmed to output an analog voltage in proportion to distance. The analog voltage is input to the Arduino microcontroller's A/D converter and converted to distance with a calibration equation supplied by the manufacturer. Infrared sensors such as the GP2 series (Sharp Electronics Corporation, Mahwah, NJ USA) offer a variety of operating ranges.

\subsubsection{Pressure}

Maintaining proper pressure and measuring the existing pressure are important in many processes and environments. Atmospheric air pressure is an important meteorological parameter, for example, and liquid pressure can be used to determine fluid depth based on hydrostatic pressure relationships.

Many pressure sensing devices are available and range from simple sensing elements to amplified, calibrated, and temperature-compensated sensors. Sensing configu- rations typically consist of piezoresistive elements and a silicon diaphragm arranged in a Wheatstone-bridge circuit. A change in pressure causes the diaphragm to flex and changes the resistance values of the piezoresistive elements. Since changes are very small, the change in electrical output of the Wheatstone bridge is also small, requiring accurate voltage-measuring circuitry. Amplifying the output signal allows the signal to be measured with an A/D converter on the Arduino. Temperature changes can also affect the piezoresistive elements, resulting in the need for temperature compensation under conditions of large temperature swings. A range of pressure sensors, including the non-temperature-compensated 24PC, temperature-compensated 26PC, and fully compensated and amplified 40PC series (Honeywell Sensing and Control, Golden Valley, MN USA) can be interfaced and read with the Arduino's microcontroller.

\subsubsection{Resolution of Analog Sensor Measurements}

Analog sensors output a voltage signal which is converted into a numerical value by an $\mathrm{A} / \mathrm{D}$ converter. The $\mathrm{A} / \mathrm{D}$ converter is characterized by a known, reference voltage, which determines the range of acceptable voltage signals, and the number of digital values, or bits, into which the voltage range is divided. The Arduino's microcontroller contains a 10-bit A/D converter, meaning that the voltage range is divided into $2^{10}$, or 1024 , divisions. To measure a sensor's voltage signal, the A/D converter compares the voltage level to the reference voltage, and returns a proportional digital value in the range of 0 to 1023 .

The A/D converter characteristics determine the resolution and accuracy of voltage measurements. The resolution, or smallest change in voltage that the $\mathrm{A} / \mathrm{D}$ converter can detect, is dependent on the $\mathrm{A} / \mathrm{D}$ converter's number of bits and the reference voltage. The Arduino's microcontroller has a built-in $1.1 \mathrm{~V}$ reference, which provides the $\mathrm{A} / \mathrm{D}$ converter with a resolution of $1.1 \mathrm{~V} /$ 1024 bits, or $0.00107 \mathrm{~V} /$ bit. The microcontroller's 5-V power supply voltage can also be used as a reference, resulting in an $\mathrm{A} / \mathrm{D}$ conversion resolution of 0.00488 $\mathrm{V} / \mathrm{bit}$

Resolution can be increased or decreased by changing the number of A/D conversion bits. External A/D converter chips are available which have higher-bit resolutions and can be easily interfaced with the Arduino. The MCP3424 (Microchip Technology Inc., Chandler, AZ USA) is an A/D converter chip which can read four input voltage signals with 18-bit (262,144 divisions) resolution. With a 5-V reference voltage, this would provide an A/D resolution of $0.0000191 \mathrm{~V} / \mathrm{bit}$. The MCP3424 communicates with the microcontroller using I2C.

To illustrate the effect of $\mathrm{A} / \mathrm{D}$ converter resolution on sensor measurements, consider an analog temperature 
sensor that outputs a voltage signal between 0 and $5 \mathrm{~V}$ over a temperature range of 0 to $65 \mathrm{C}$. The signal, therefore, changes by $65 \mathrm{C} / 5 \mathrm{~V}$, or $13 \mathrm{C} / \mathrm{V}$. Using the microcontroller's built-in 10-bit A/D converter and a $5-\mathrm{V}$ reference, with a resolution of $0.00488 \mathrm{~V} / \mathrm{bit}$, the resolution of temperature measurements would be $13 \mathrm{C} / \mathrm{V} * 0.00488$ $\mathrm{V} /$ bit, or $0.06 \mathrm{C} / \mathrm{bit}$, which would be acceptable for most applications.

The resolution of a signal from a non-amplified pressure sensor, with an output of 0 to $10 \mathrm{mV}$ over a range of 0 to $100 \mathrm{kPa}$, would have a measurement resolution of $100 \mathrm{kPa} / 0.01 \mathrm{~V}^{*} 0.00488 \mathrm{~V} / \mathrm{bit}$, or $48.8 \mathrm{kPa} / \mathrm{bit}$. This would be unacceptable, providing only three measurements $(0,48.8$, and $97.6 \mathrm{kPa})$ over the entire measurement range. Using the MCP3424 external A/D converter, with 18-bit resolution, would greatly improve voltagemeasurement capability and provide a pressure-measurement resolution of $0.038 \mathrm{kPa} / \mathrm{bit}$.

\subsection{Time-Keeping}

In many data-collection efforts, proper timing of measurements and date- and time-stamping of sensor data are required. The microcontroller on the Arduino board has a very accurate $16 \mathrm{MHz}$ oscillator and the ability to measure time increments with microsecond accuracy, but is not designed to provide real time (hours, minutes) and date information. If electrical power to the microcontroller is lost, the oscillator and microcontroller program cease to function, and any timing information is also lost.

External real-time clock (RTC) chips are used to provide time-keeping functions, with dedicated built-in or added backup batteries to retain accurate time information. RTCs such as the DS1307 and DS1337 (Maxim Integrated Products, Inc., Sunnyvale, CA USA) interface with the microcontroller using the I2C protocol, while others, such as the MCP795 (Microchip Technology Inc., Chandler, AZ USA) communicate via SPI. Simple routines in the microcontroller program access the RTCs to set or read time and date information, which can then be used to trigger sensor measurements at regular time intervals or record timing information of events.

\subsection{Data Storage}

Data collection often involves long-term, automated storage of sensor measurements. While the Arduino's microcontroller has extensive memory available for program storage, non-volatile data-storage capability is limited. On-board memory consists of $1 \mathrm{~kb}$ (1000 bytes), so a maximum of 1000 data values could be stored and retained if battery power were interrupted. To expand the storage capacity, external storage must be added.

External memory chips are available with varying amounts of non-volatile memory. The 24LC family of memory chips (Microchip Technology Inc., Chandler, AZ USA), for example, are available in capacities from 16 bytes to $65,356 \mathrm{~KB}$. These chips communicate via the $\mathrm{I} 2 \mathrm{C}$ protocol and have individual identification numbers so that multiple chips could be connected to increase storage amounts considerably.

For permanent or large-capacity storage, add-on boards are available which provide data storage to standard SD memory cards (Adafruit Industries, New York, NY USA) or microSD memory cards (Sparkfun Electronics, Boulder, CO USA). Memory cards are commonly available with storage capacities from 1 gigabyte (GB) to several GB, are inexpensive, and can be easily interfaced with the Arduino hardware. Since the memory cards can be read with a computer, data can be transferred quickly and easily between datalogger and computer. Software libraries have been written to provide all memory card reading, writing, and data-access functions, enabling rapid incorporation of memory-card storage into a datalogging project.

\section{Sensing Applications}

To illustrate how the Arduino platform can be used to develop and implement an inexpensive, automated data collection and monitoring program, several examples are presented. These examples include a brief description of the circuitry and details of the project implementation. Microcontroller programs are not included but are freely available by contacting the authors.

\subsection{Soil-Moisture Monitoring Datalogger}

Monitoring moisture status of the soil profile is useful in scheduling irrigations and monitoring the movement or availability of water in the soil profile. Sensors are installed in the soil profile at various depths within a crop's root zone and are monitored periodically. A datalogger was designed to record measurements from three soilmoisture sensors at one-hour intervals, and store the measurements, along with the date and time, to a microSD memory card.

\subsubsection{Hardware}

The main components of the datalogger include an Arduino-compatible microcontroller board, voltage regulator, microSD/prototyping shield, and real-time clock/ calendar. The Diavolino microcontroller board (Evil Mad Science LLC, Sunnyvale, CA USA) was chosen for its low cost, simple and low-component design, and ease of modification for battery-powered operation. The board, designed to operate from the $5 \mathrm{~V}$ power supplied via the USB computer connection, was modified by adding a two-pin header to connect an external AA battery pack. An LP2950 voltage regulator (National Semiconductor 
Corp., Santa Clara, CA USA) and capacitors were added to convert the unregulated battery voltage to a stable $5-\mathrm{V}$ source to power the microcontroller. A trace on the printed circuit board, which powered the board from the USB connection, was then cut so that the only power source was the AA battery pack. The modified Diavolino microcontroller board is shown in Figure 1.

The microSD shield (Sparkfun Electronics, Boulder, CO USA) consists of a microSD-card holder, with on-board voltage-level shifter to supply the proper voltage levels for reading from and writing to a microSD card, and a prototyping area to incorporate additional circuitry into the shield. The microSD shield was designed to be powered from the microcontroller board's power supply, thus the microSD card and voltage-level shifter would always be powered and continuously drawing current. The shield was modified for battery-powered operation by rerouting the power supply for the microSD card and voltage-level shifter to one of the microcontroller's digital pins so that the components could be turned on and off as needed. A microSD card (Samsung) with a 2 gigabyte storage capacity was then inserted into the microSD card holder.

A circuit was designed and added to the microSD shield's prototyping area to measure the output from three soil-moisture sensors. A DS1337 real-time clock/ calendar chip provides date and time information for the microcontroller to make sensor readings at regular time intervals and to date- and time-stamp sensor data stored to the microSD card. A $32.768 \mathrm{kHz}$ crystal oscillator provides an accurate timing signal for the DS1337, and a 3.3-V lithium coin cell battery powers the clock chip. The DS1337 interfaces with the microcontroller via the I2C protocol.

The soil-moisture sensors consist of three Watermark 200SS matric-potential sensors whose electrical resistance varies with moisture content. A circuit was designed in which each sensor, which acts as a variable

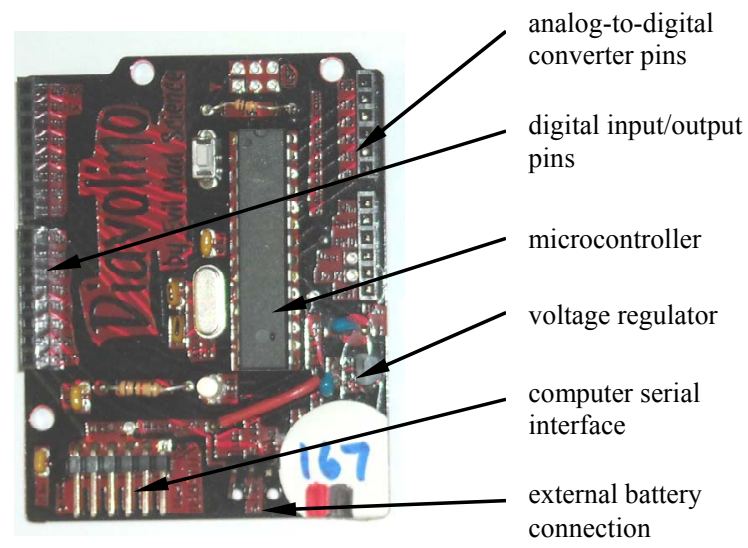

Figure 1. Modified Diavolino Arduino-compatible microcontroller board. resistor, forms one leg of a half bridge, or voltage divider. The half bridge is connected to two digital pins on the microcontroller, and each voltage-divider output is connected to an $\mathrm{A} / \mathrm{D}$ pin. A photograph of the completed circuit, mounted on the microSD shield, is shown in Figure 2, and a schematic of the circuit is shown in Figure 3. A list of materials, with sources and approximate cost (small-quantity retail price, in the United States, US dollars, 2011), is provided in Table 1.

\subsubsection{Software}

Using the Arduino IDE installed on a personal computer, a microcontroller program, called a sketch on the Arduino platform, was written to read the real-time clock, make soil-moisture sensor measurements, and store the time and sensor data to a microSD card. Communication between the computer and microcontroller board requires an RS-232 serial connection, which was accomplished via an FTDI USB-serial cable, which interfaces to the

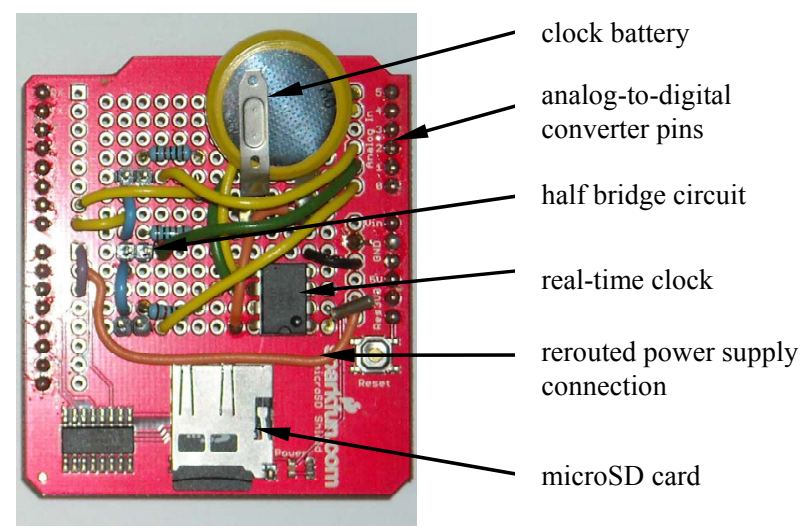

Figure 2. Modified microSD shield with circuit components installed.

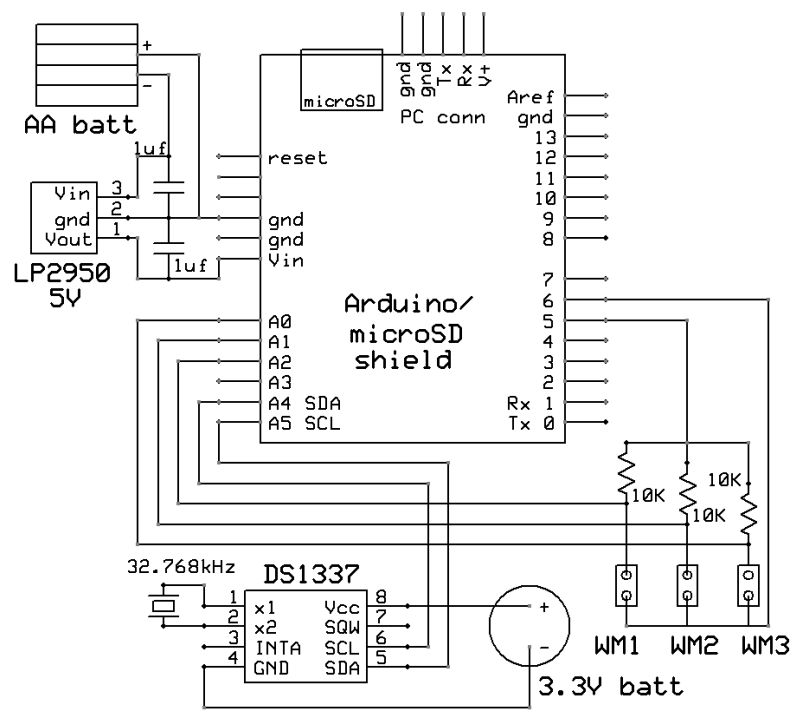

Figure 3. Schematic for soil-moisture sensor datalogger. 
Table 1. List of materials for soil-moisture sensor datalogger.

\begin{tabular}{|c|c|c|c|}
\hline Main components & Part number & Supplier & Cost $\$$ \\
\hline Microcontroller board & Diavolino & Evil Mad Science & 13 \\
\hline microSD card & $2 \mathrm{gb}$ & Samsung & 4 \\
\hline Real-time clock/calendar & DS1337 & Maxim Integrated Products & 3 \\
\hline Oscillator & $32.768 \mathrm{kHz}$ & Citizen America & 1 \\
\hline \multirow[t]{3}{*}{ Regulator } & LP2950 5V & National Semiconductor & 2 \\
\hline & \multicolumn{2}{|c|}{ Miscellaneous (capacitors, resistors, headers, batteries) } & 7 \\
\hline & \multicolumn{2}{|c|}{ Datalogger Total } & 44 \\
\hline
\end{tabular}

computer's USB hub and creates a virtual serial port.

To enable long-term, battery-powered operation of the datalogger, the microcontroller was programmed to spend most of its time in a low-power, sleep mode. Periodically, the microcontroller would wake up and read the current time from the real-time clock. If it was time to take a measurement, the microcontroller would power the measurement circuit, otherwise it would go back to sleep. At one-hour intervals, the measurement circuit on the microSD shield was enabled, and the soil-moisture sensors were read and data stored to the microSD card.

To properly read a Watermark 200SS sensor, an alternating current source is recommended in order to avoid polarizing the sensor with a prolonged direct-current excitation, which can influence sensor measurements and degrade the sensor over time. The microcontroller can only supply a direct-current excitation, however, so a pseudo-alternating current source was created by rapidly switching the polarity of the direct-current voltage sent to power the sensor, and the sensors were then read under each polarity. Digital pin 6 was first set high (a voltage level of $5 \mathrm{~V}$ ) and pin 5 was set low (a voltage level of 0 $\mathrm{V})$ so that current flowed through the half bridge in one direction (see Figure 3). The output voltage, Vout, between the 10 kohm resister, $\mathrm{R}$, and the Watermark sensor, Rwm, was measured with an A/D converter, and the sensor resistance was calculated using the voltage-divider relationship, Vout $=\mathrm{R} /(\mathrm{Rwm}+\mathrm{R}) * 5 \mathrm{~V}$. The polarity of the half bridge was then switched by setting pin 5 high and pin 6 low, so that current flowed in the opposite direction, and output voltage was again measured and sensor resistance calculated. This was repeated five times, and an average resistance was calculated.

To arrive at the sensor's final output, namely the matric-potential of the soil, in $\mathrm{kPa}$, a calibration equation is required to convert sensor resistance to matric potential. Much work has been done calibrating and verifying the
Watermark 200SS sensor [14-16], and several calibration equations have been proposed. The equation of Shock et al. [16] was chosen, written as SWP $=(4.093+3.213$ Rwm $) /(1-0.009733 *$ Rwm $-0.01205 *$ Tsoil $)$, where SWP is the soil-water potential $(\mathrm{kPa}), \mathrm{Rwm}$ is the sensor resistance (ohms), and Tsoil is the soil temperature $\left({ }^{\circ} \mathrm{C}\right)$. While sensor performance has been shown to vary slightly with temperature, and a temperature-correction factor is included in the calibration equation, soil temperature was not measured and, instead, a constant temperature of $25{ }^{\circ} \mathrm{C}$ was used. To improve accuracy of sensor readings, a soil-temperature sensor could be added to the datalogger circuit and actual temperature measurements input to the calibration equation.

Following sensor measurements, power was sent to the microSD card circuit, and the data were stored to the microSD card. Data were stored as ASCII text, separated by spaces, in a plain text file, and consisted of six values; a datalogger board identification number, date (month/ day/year), time of day (hour), sensor \#1 reading $(\mathrm{kPa})$, sensor \#2 reading $(\mathrm{kPa})$, and sensor \#3 reading $(\mathrm{kPa})$. The microcontroller then turned all power off to the microSD shield and returned to low-power, sleep mode.

\subsubsection{Data}

Thirty soil-moisture sensor dataloggers were constructed and deployed to monitor soil-moisture status in experimental research plots at the USDA Agricultural Research Service's Jamie Whitten Delta States Research Center at Stoneville, Mississippi USA. Research plots planted to soybean and cotton were instrumented with soil-moisture sensors and Arduino-based dataloggers. At each instrumented site, Watermark sensors were installed at three depths; 15-, 30-, and 60-cm, below the soil surface. The sensors were connected to a datalogger, and the datalogger was placed inside a weatherproof plastic enclosure attached to a wooden stake driven into the ground. The 
datalogger was turned on, and collected sensor data at one-hour intervals throughout the entire growing season. Periodically, each site was visited to download data from the microSD card to a portable tablet computer. The text data files were then returned to the office, uploaded to a desktop computer, and input to a spreadsheet for analysis and viewing. Typical data, from one site over a sevenweek period following planting in 2011, are shown in Figure 4.

Soil-water potential values near 0 indicate very moist soil conditions, with soil-water levels decreasing as the water-potential values become more negative. Hourly data from the three sensors were input to a spreadsheet, and the average of the three sensor readings was calculated. The average values were used to determine when an irrigation was needed. When the average values reached a threshold value of $-50 \mathrm{kPa}$, an irrigation was scheduled. In Figure 4, soil-water levels decreased early in the season as the growing crop extracted water until rainfall occurred on 6/21, rewetting the soil. As soil water was used by the crop, the levels dropped until reaching $-50 \mathrm{kPa}$, and two irrigations were required. Evident in the data are differences in water use with depth in the soil profile. Early in the season, changes in water potential were slower at $30 \mathrm{~cm}$ than at $15 \mathrm{~cm}$, and much slower at $60 \mathrm{~cm}$, suggesting more active roots in the shallower depths. As the season progressed, water-use rates increased at the $30-\mathrm{cm}$ depth, and later at the $60-\mathrm{cm}$ depth, suggesting increases in root activity and water extraction.

\subsection{Ultrasonic Water-Level Datalogger}

Fluid levels are measured in a variety of applications; fuel tanks, water reservoirs, and irrigation canals, for example. Evaporation pans are used to estimate the evaporative demands of the atmosphere in order to determine crop water use and soil evaporation rates for input in water-balance and evapotranspiration studies, and to assist in irrigation scheduling. A datalogger was de-

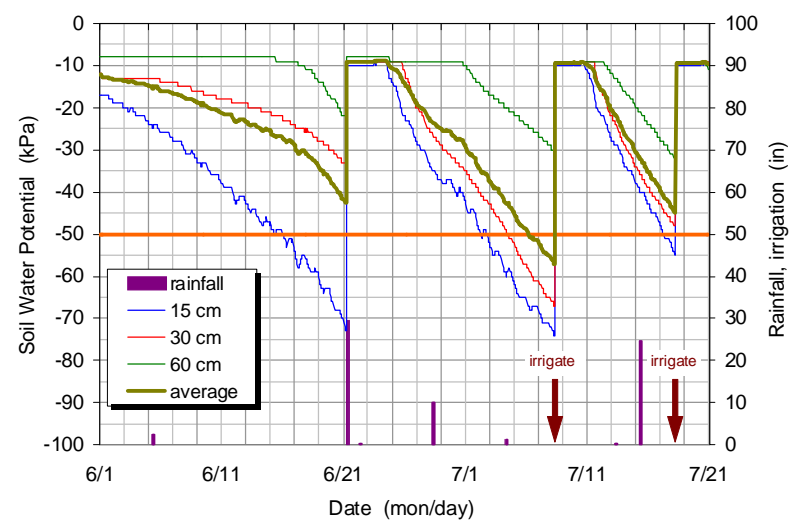

Figure 4. Data collected with the soil-moisture datalogger for a seven-week period in 2011. veloped to automate the measurement of the depth of water in an evaporation pan using an ultrasonic distance sensor.

\subsubsection{Hardware}

The ultrasonic water-level datalogger circuit is based on that of the soil-moisture datalogger, and incorporates many of the same circuit components. The same Ardinocompatible microcontroller board was used, and was modified in the same manner to supply a stable power source and enable battery-powered operation. The same microSD/prototyping shield and real-time clock components were also used. A schematic of the ultrasonic water level datalogger is shown in Figure 5.

An ultrasonic distance sensor, model SRF-04 (Devantech Ltd., Norfolk, UK), interfaces with the microcontroller via three digital pins; power, trigger, and echo pulse. The sensor consists of two ultrasonic transducers, one to send an ultrasonic pulse and one to receive the pulse's echo. To make a measurement, power is supplied to the sensor, and a measurement is initiated by sending a brief signal to the trigger pin, which causes an ultrasonic pulse to be sent. The microcontroller then begins monitoring the echo pulse pin, and measures the length of time it takes to receive an echo signal.

A temperature sensor was added to measure the air temperature of the environment. The LM35 analog temperature sensor outputs an analog-voltage signal in proportion to its temperature. The signal is input to one of the microcontroller's A/D converters, and a calibration equation supplied by the manufacturer is used to convert the voltage signal to temperature.

\subsubsection{Software}

The microcontroller program for the ultrasonic waterlevel datalogger used many of the same routines written for the soil-moisture datalogger. The microcontroller

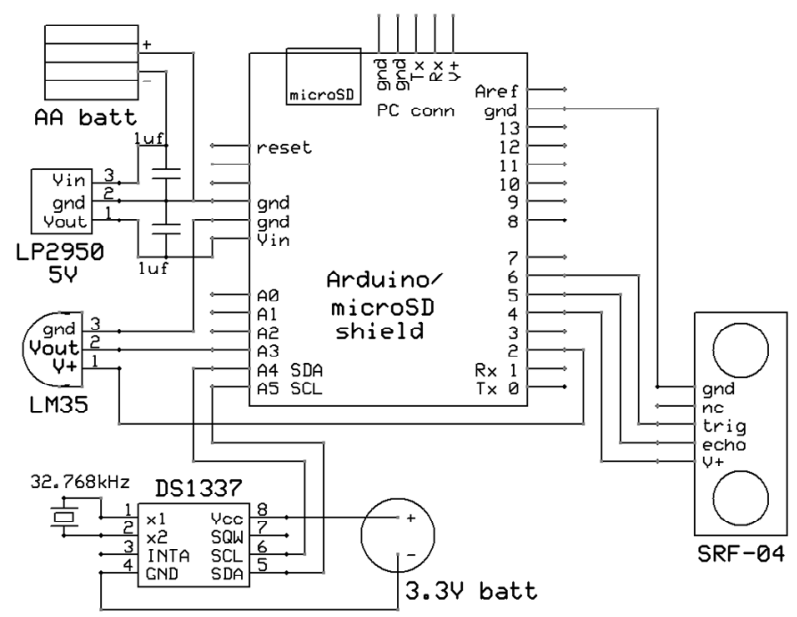

Figure 5. Schematic for ultrasonic water-level datalogger. 
wakes periodically from a low-power sleep mode to read the real-time clock and determine if it is time to take measurements. If so, measurements are taken and recorded, otherwise, the microcontroller goes back to sleep.

At each measurement interval, the microcontroller turns on the temperature sensor and makes an air temperature measurement. The ultrasonic sensor is then powered, a trigger signal is sent, and the time for an echo signal to return is measured. The time for the echo to return is then used to calculate the distance, based on the speed of sound, between the sensor and the surface upon which the ultrasonic pulse impacted. The speed of sound, however, is strongly dependent on the air temperature, and slightly affected by humidity [17], and can be corrected to improve the accuracy of distance measurements using the relationship $\mathrm{v}=331 \mathrm{~m} / \mathrm{s}+0.6 \mathrm{~m} / \mathrm{s} / \mathrm{C}^{*} \mathrm{~T}$, where $\mathrm{v}$ is the speed of sound $(\mathrm{m} / \mathrm{s})$ and $\mathrm{T}$ is the air temperature $\left({ }^{\circ} \mathrm{C}\right)$. To make a distance measurement, the air temperature measurement is first used to correct the speed of sound value. The speed of sound and the time taken to return the pulse echo are then used to calculate the distance from the sensor to the reflecting surface. This distance is then subtracted from the distance of the sensor to the bottom of the evaporation pan, measured previously when installing the ultrasonic sensor, to determine the depth of water in the pan.

Following air temperature and water level measurements, the data, microcontroller board identification number, and date and time are written to the microSD card. The microcontroller then turns off power to the circuit and returns to low-power, sleep mode.

\subsubsection{Data}

Two ultrasonic water-level dataloggers were constructed and installed in summer 2011 and operated for a threemonth period. The sensors were installed on an evaporation pan approximately $300 \mathrm{~mm}$ above the bottom of the pan. Sensor measurements were recorded at one-hour intervals, and the data were periodically downloaded from the microSD card during periodic site visits. During site visits, manual measurements of the water level were made by inserting a steel ruler into the evaporation pan and reading the depth of water. The depth of water in the pan varied between 70 and $195 \mathrm{~mm}$, decreasing as water evaporated in response to the environmental demand and increasing due to rainfall and periodic manual refilling.

Data collected during a four-day period with one ultrasonic sensor datalogger are shown in Figure 6. Data include air temperature, raw depth (before correcting the speed of sound for temperature) and temperature-corrected depth, and manual measurements of the water levels. Large increases in apparent depth of water can be seen in the raw sensor readings each morning beginning around 6:00, as the sun rose and air temperature in- creased rapidly. The raw depths also continued to appear to decrease after sunset, when evaporation would be expected to cease. Correcting the speed of sound for air temperature mostly eliminates these errors, resulting in expected changes in water level, decreasing during daylight hours and minimal changes during nighttime. An increase in depth can be seen in response to a manual addition of water to the pan.

Accuracy of ultrasonic measurements was determined by comparing water levels measured with the ultrasonic sensors to those measured manually. Manual depth measurements were made 18 times, at varying times throughout the three-month period and at varying times of day. Manually measured water levels ranged from 75 to $158 \mathrm{~mm}$. Comparison of measurements from the two ultrasonic sensors is shown in Figure 7, and indicates a very good agreement with the manual measurements, with a standard error of measurements of approximately $2 \mathrm{~mm}$.

\subsection{Environmental Datalogger}

An Arduino-based datalogger can also be built using a

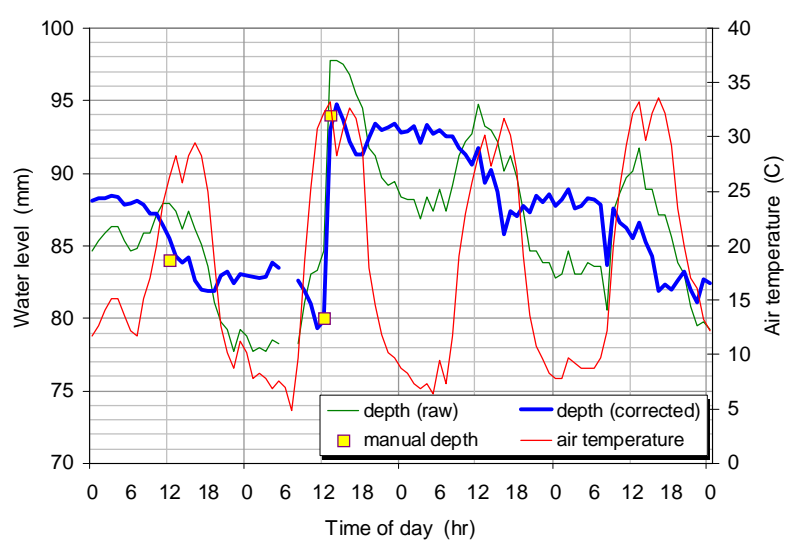

Figure 6. Hourly data collected with one ultrasonic water-level datalogger during a four-day period.

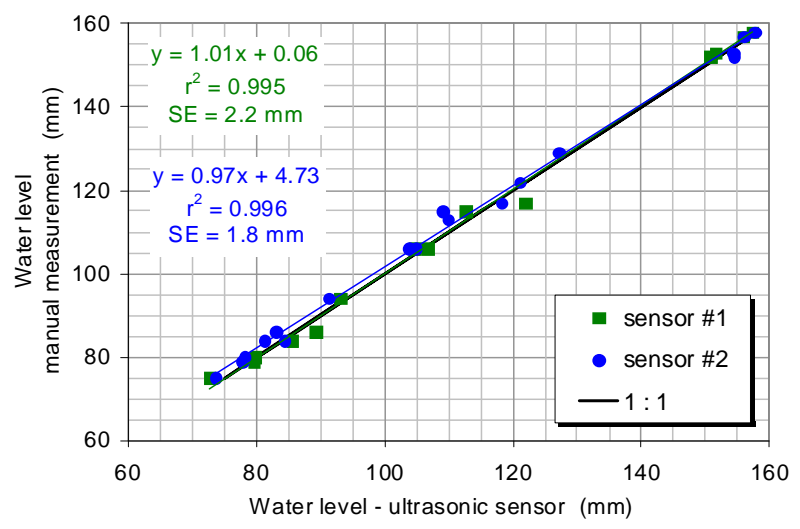

Figure 7. Comparison of manual versus automated measurements for two ultrasonic water-level dataloggers. 
custom printed circuit board (PCB) rather than starting with a commercially available board. A datalogger was designed and fabricated to collect environmental data in a forested setting. The datalogger was de signed to accommodate a variety of sensor types, but was primarily intended to measure soil moisture and air temperature. Rather than developing a system around a commercially available Arduino board, a custom PCB was created which contained the Arduino microcontroller and other components.

\subsubsection{Custom Circuit-Board Design}

Creating a new PCB requires some additional skills but is a viable approach for many users. The main advantages of creating a custom PCB include the selection of specific components for the particular project, the creation of a board with a custom layout or size/configuration, and the reduction, in some cases, in total cost.

The minimum components needed to create an Arduino board include the ATmega328 microcontroller, a resonator (self-contained oscillator circuit), a reset button, a voltage regulator, connectors for a battery pack, computer interface, and a few resistors and capacitors. ATmega328 microcontroller chips are available preprogrammed with the Arduino-system's bootloader, enabling the use of the Arduino IDE to create and upload programs to the microcontroller. Additional components for most datalogger projects include a real-time clock/ calendar, a memory device, and one or more light-emitting diodes (LEDs) to indicate the operational status of the datalogger. All of these components are readily available as through-hole components which can be soldered to the PCB with a soldering iron.

The process of creating a custom circuit board begins with circuit and PCB design. Several software packages are available, some in freely available, open-source versions, to design the electrical schematic and then lay out the circuit on a PCB. A graphical user interface simplifies design, and the software creates a set of files in formats standardized for PCB manufacturing, which can then be transmitted to a PCB manufacturer. The manufacturer produces the bare $\mathrm{PCB}$, and the final board is constructed by soldering the components to the PCB by hand.

\subsubsection{Hardware}

A board was designed using the freely available Design-Spark PCB software (www.designspark.com/pcb). The circuit was designed using a graphical schematic view, in which connections between circuit components are created but the actual size, shape, and layout of the components are unimportant. This schematic is then transferred to a printed circuit board layout, where the software suggests the physical layout and connecting traces of the components. The user is able to modify the layout as desired, to create a PCB that is easy to assemble, or which fits certain dimensional or other constraints.

The resulting board design, with dimensions of approximately $60 \times 90 \mathrm{~mm}$, was then electronically transmitted for fabrication using SeeedStudio's Fusion PCB service (www.seeedstudio.com/propagate). Dataloggers were assembled by soldering circuit components to the custom PCB, with each datalogger requiring approximately 20 minutes to complete. A list, with approximate cost of components, excluding sensors, is provided in Table 2. The original design layout is shown in Figure 8(a), with resulting bare printed circuit board and finished datalogger board shown in Figures 8(b) and 8(c), respectively.

Dataloggers were deployed in the field along with air-temperature and soil-moisture sensors. Air temperature was measured using a DS18B20 12-bit digital temperature sensor (Maxim Integrated Products, Inc., Sunnyvale, CA USA). The sensor uses the 1-Wire communication protocol to transfer measurements to the microcontroller, and contains an internal 18-bit A/D converter which provides temperature measurements with a resolution of $0.06 \mathrm{C}$. Soil-moisture measurements were made using an EC-20 capacitive sensor. The microcontroller provided an excitation voltage to power the sensor via a digital IO pin, and measured the analog output voltage with a built-in $\mathrm{A} / \mathrm{D}$ converter.

A battery pack consisting of 5 AA alkaline batteries enabled long-term remote operation by ensuring adequate voltage as the batteries discharged.

\section{Summary}

Advances in electronic technologies, microcontrollers, and sensors offer researchers a variety of new and inex-

Table 2. List of materials for environmental datalogger.

\begin{tabular}{cccc}
\hline Main components & Part number & Supplier & Cost \$ \\
\hline $\begin{array}{c}\text { Microcontroller with } \\
\text { bootloader }\end{array}$ & ATmega328 & $\begin{array}{c}\text { Sparkfun } \\
\text { Electronics }\end{array}$ & 6 \\
Printed circuit board & SeeedStudios & 3 \\
Memory chip & 24LC512 & Microchip & 4 \\
$\begin{array}{c}\text { Real-time } \\
\text { clock/calendar }\end{array}$ & DS1307 & $\begin{array}{c}\text { Maxim Integrated } \\
\text { Products }\end{array}$ & 1 \\
Oscillator & $32.768 \mathrm{kHz}$ & $\begin{array}{c}\text { Citizen America } \\
\text { National }\end{array}$ & 1 \\
Regulator & LP2950 & $\begin{array}{c}\text { Semiconductor } \\
\text { Screw terminals }\end{array}$ & 1 \\
Miscellaneous (capacitors, resistors, connectors, LEDs) & 6 \\
\multicolumn{3}{c}{ Datalogger Total } \\
\hline
\end{tabular}


(a)

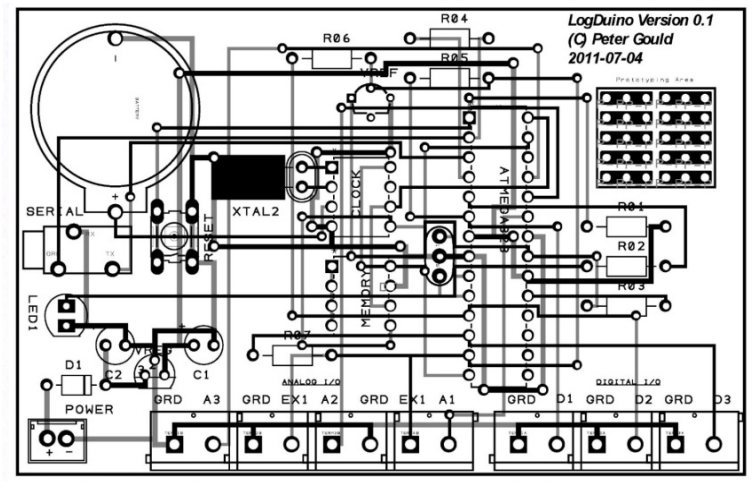

(b)

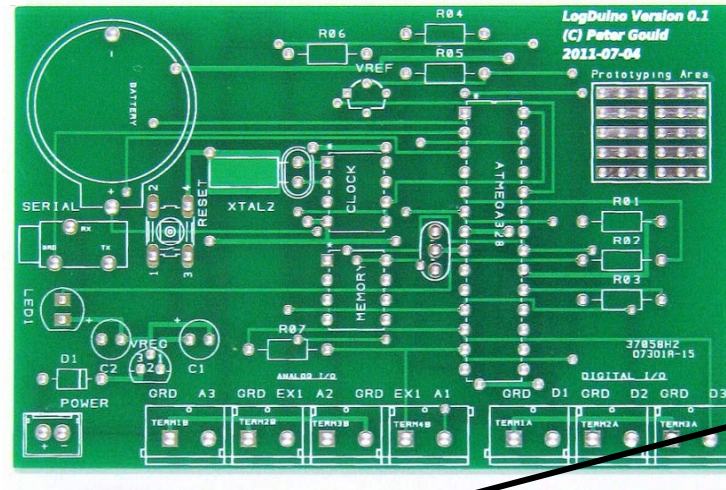

(c)

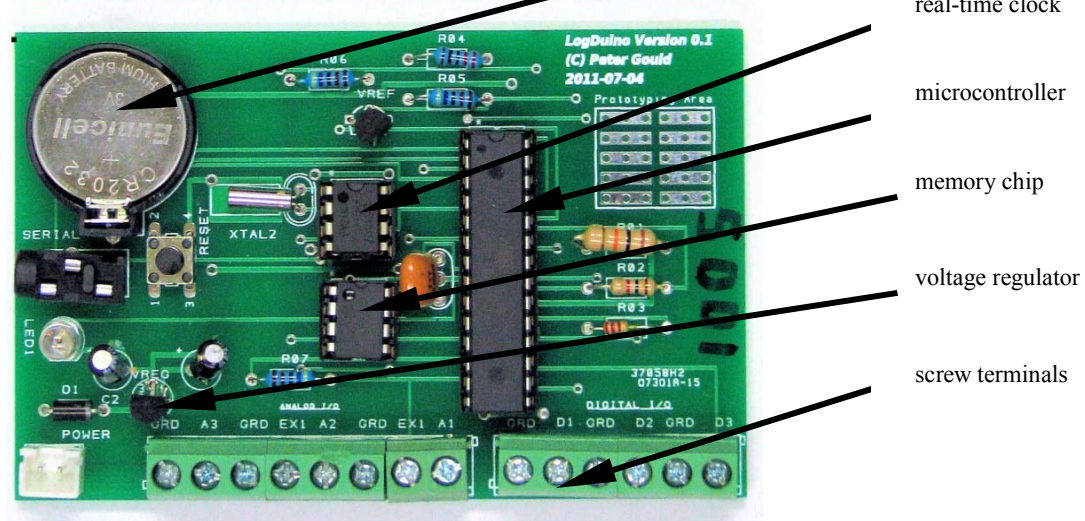

Figure 8. Arduino-based datalogger fabricated on a custom printed circuit board: top, (a) circuit-board layout; middle; (b) bare circuit board; bottom; (c) completed board with components installed.

pensive sensing, monitoring, and control capabilities The concept of open-source hardware, in which hardware designs, software programs, and development efforts are made freely available to all, help facilitate and expand the adoption of these capabilities. The open-source hardware Arduino development platform has great potential for implementation in scientific research applications, and can empower researchers with flexible, inexpensive tools for expanding their data-collection, automation, and control capabilities.

\section{Disclaimer}

Mention of a trade name, proprietary product, or specific equipment does not constitute a guarantee or warranty by the United States Department of Agriculture, and does not imply approval of the product to the exclusion of others that may be available.

\section{REFERENCES}

[1] F. H. Moody, J. B. Wilkerson, W. E. Hart and N. D. Sewell, "A Digital Event Recorder for Mapping Field Operations," Applied Engineering in Agriculture, Vol. 20, No. 1, 2004, pp. 119-128.

[2] K. A. Noordin, C. C. Onn and M. F. Ismail, "A Low-Cost Microcontroller-Based Weather Monitoring System," CMU Journal, Vol. 5, No. 1, 2006, pp. 33-39.

[3] D. K. Fisher, "Automated Collection of Soil-Moisture Data with a Low-Cost Microcontroller Circuit," Applied 
Engineering in Agriculture, Vol. 23, No. 4, 2007, pp. 493-500.

[4] G. Vellidis, M. Tucker, C. Perry, C. Kvien and C. Bednarz, "A Real-Time Wireless Smart Sensor Array for Scheduling Irrigation," Computers and Electronics in Agriculture, Vol. 61, No. 1, 2008, pp. 44-50. doi:10.1016/j.compag.2007.05.009

[5] D. K. Fisher and H. Kebede, "A Low-Cost Microcontroller-Based System to Monitor Crop Temperature and Water Status," Computers and Electronics in Agriculture, Vol. 74, No. 1, 2010, pp. 168-173. doi:10.1016/j.compag.2010.07.006

[6] Arduino, "An Open-Source Electronics Prototyping Platform," 2012. http://www.arduino.cc

[7] D. Bri, H. Coll, M. Garcia and J. Lloret, "A Multisensor Proposal for Wireless Sensor Networks," 2nd International Conference on Sensor Technologies and Applications, Cap Esterel, 25-31 August 2008, pp. 270-275.

[8] L. Buechley and M. Eisenberg, "The LilyPad Arduino: Toward Wearable Engineering for Everyone," Pervasive Computing, Vol. 7, No. 2, 2008, pp. 12-15. doi:10.1109/MPRV.2008.38

[9] J. Zhang, S. K. Ong and A. Y. C. Nee, "Design and Development of a Navigation Assistance System for Visually Impaired Individuals," Proceedings of the 3rd International Convention on Rehabilitation Engineering \& Assistive Technology, Singapore, 22-26 April 2009.

[10] N. W. Bergmann, M. Wallace and E. Calia, "Low Cost Prototyping System for Sensor Networks," 6th International Conference on Intelligent Sensors, Sensor Networks and Information Processing, Brisbane, 7-10 De- cember 2010, pp. 19-24. doi:10.1109/ISSNIP.2010.5706802

[11] D. Gordon, M. Beigl and M. A. Neumann, "Dinam: A Wireless Sensor Network Concept and Platform for Rapid Development," 7th International Conference on Networked Sensing Systems (INSS), Kassel, 15-18 June 2010, pp. 57-60. doi:10.1109/INSS.2010.5573290

[12] J. Sarik and I. Kymissis, "Lab Kits Using the Arduino Prototyping Platform," Frontiers in Education Conference, Washington DC, 27-30 October 2010, pp. 1-5.

[13] A. M. Thomas, "In situ Measurement of Moisture in Soil and Similar Substances by 'Fringe' Capacitance," Journal of Scientific Instrumentation, Vol. 43, No. 1, 1966, pp. 21-27. doi:10.1088/0950-7671/43/1/306

[14] S. J. Thomson and C. F. Armstrong, "Calibration of the Watermark Model 200 Soil Moisture Sensor," Applied Engineering in Agriculture, Vol. 3, No. 2, 1987, pp. 186189.

[15] E. P. Eldredge, C. C. Shock and T. D. Stieber, "Calibration of Granular Matrix Sensors for Irrigation Management," Agronomy Journal, Vol. 85, No. 6, 1993, pp. 1228-1232.doi:10.2134/agronj1993.00021962008500060 $\underline{025 \mathrm{x}}$

[16] C. C. Shock, J. M. Barnum and M. Seddigh, "Calibration of Watermark Soil Moisture Sensors for Irrigation Management," Proceedings of the International Irrigation Show, San Diego, 1-3 November 1998, pp. 139-146.

[17] D. A. Bohn, "Environmental Effects on the Speed of Sound," Journal of the Audio Engineering Society, Vol. 36, No. 4, 1988, pp. 223-231. 